\title{
Familias y Docentes: Garantes del Aprendizaje durante el Confinamiento
}

\section{Families and Teachers: Guarantees of Learning during Confinement}

\author{
David Hortigüela-Alcalá ${ }^{1}$ \\ Ángel Pérez-Pueyo ${ }^{2}$ \\ Mercedes López-Aguado ${ }^{2}$ \\ Jesús Manso-Ayuso ${ }^{3}$ \\ Javier Fernández-Río ${ }^{4}$ \\ ${ }^{1}$ Universidad de Burgos, España \\ ${ }^{2}$ Universidad de León, España \\ ${ }^{3}$ Universidad Autónoma de Madrid, España \\ ${ }^{4}$ Universidad de Oviedo, España
}

\begin{abstract}
El cierre de los centros educativos en España ha supuesto una adaptación inmediata sobre la forma de garantizar el aprendizaje. Los docentes han tenido que adaptarse de manera inmediata a un entorno digital de enseñanza y las familias han debido de atender con garantías a las tareas virtuales de sus hijos. El presente estudio tiene dos objetivos: a) analizar las variables relativas a la relación existente entre la familia, los centros educativos y los docentes, así como sus problemas para apoyar a los alumnos; b) analizar el efecto que tiene el modelo de enseñanza empleado por el docente en el seguimiento de la enseñanza virtual. Se ha utilizado una metodología cuantitativa con un diseño ex post facto descriptivo por encuesta, en el que han participado 3700 docentes y 5867 familias de todo el territorio español. Las principales dificultades se han encontrado en la falta de apoyo por parte de la administración y en la formación en competencia digital del profesorado. También ha quedado de manifiesto la necesidad de trabajar la autonomía y la autorregulación de los estudiantes y además existieron deferencias significativas en función del modelo de enseñanza (centrado en contenido versus en competencia) de los docentes.
\end{abstract}

Descriptores: Modelos de enseñanza; Familias; Docentes; Desigualdad educativa; Escuela en casa; Covid-19.

The closure of schools in Spain has meant an immediate adaptation on how to guarantee learning. Teachers have had to adapt immediately to a digital teaching environment and families have had to deal with their children's virtual tasks with guarantees. This study has two objectives: a) to analyze the variables related to the relationship between the family, the schools and the teachers, as well as their problems in supporting the students; b) to analyze the effect that the teaching model used by the teacher has in the monitoring of virtual education. A quantitative methodology was used with an ex post facto descriptive design by survey, in which 3700 teachers and 5867 families from all over Spain participated. The main difficulties have been found in the lack of support from the administration and in the training of teachers in digital competence. The need to work on the autonomy and self-regulation of students was also highlighted, and there were also significant differences in terms of the teaching model (centred on content versus competence) of the teachers.

Keywords: Teaching methods; Families; Education right; Homeschooling; Covid19.

*Contacto: dhortiguela@ubu.es

ISSN: 2254-3139

www.rinace.net/riejs/

revistas.uam.es/riejs
Recibido: $\quad 27$ de julio 2020

$1^{\text {a }}$ Evaluación: 13 de octubre 2020

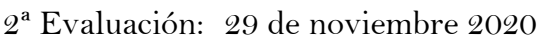

Aceptado: 16 e diciembre 2020 


\section{Introducción}

España ha conocido la primera pandemia global desde la "Peste española" (Spanish Influenza) de 1918-1920 (Fernández-Riquelme, 2020) a través de una enfermedad tan letal y desconocida como el Covid-19. El cierre de las aulas el pasado mes de marzo, como una de las formas más efectivas para frenar el avance de esta enfermedad, ha generado un verdadero shock en la sociedad española (Zubillaga y Gortazar, 2020). Tras afrontar con mayor o menor acierto la situación económica, social, política y educativa inmediata, el sistema educativo debe hacer frente a un futuro incierto a corto y medio plazo. En estas circunstancias y en un Estado descentralizado como el español, la preservación del principio constitucional de igualdad como es el derecho a la educación (Constitución Española, 1978, art. 27) requiere de las administraciones educativas una respuesta coordinada para afrontar la presente situación y así garantizar el cumplimiento de las obligaciones de los poderes públicos en esta materia (Fernández-Franco, 2020). Sin embargo, esto no solo no ha ocurrido así, sino que diferentes enfoques de las administraciones autonómicas no están consiguiendo que toda la comunidad educativa ejerza de la misma manera su derecho a la educación en condiciones de calidad y equidad, sobre todo, en los contextos más desfavorecidos (Save the Children, 2020).

La situación generada por la supresión de las clases presenciales, como consecuencia del cierre de los centros educativos, y el confinamiento de las familias ha planteado una situación inédita (Real Decreto 463/2020) en la que pues padres e hijos se han visto trabajando en el mismo contexto espacio-temporal que en muchos casos ha provocado situaciones de desventaja evidentes. Sin embargo, los docentes, generalmente también con familia, igualmente han tenido que adaptar su forma de trabajar (Díez-Gutiérrez y Gajardo-Espinoza, 2020a). De forma casi instantánea, la situación les ha obligado a modificar o transformar su manera de enseñar y de generar aprendizaje a un entorno digital, para muchos, desconocido. La consecuencia inmediata de ello ha sido que a las familias se les ha requerido su colaboración, en algunas situaciones casi en modo de codocencia, y muchas se han visto muy limitadas, por no decir imposibilitadas y/o incapacitadas, para atender a las tareas virtuales de sus hijos, no siendo suficiente con reabrir o recuperar la comunicación con el centro y los docentes.

En los siguientes apartados se va a presentar el estudio realizado comenzando por un análisis de la situación actual con un enfoque científico que nos acerque al estado de la cuestión, se presentará el método de investigación realizado, así como los resultados obtenidos. Finalizaremos con una discusión sobre los datos obtenidos, unas conclusiones, así como las debilidades, fortalezas y líneas de investigación futuras.

\section{Estado de la cuestión}

Esta pandemia, generada por el Covid-19, obliga a determinar el grado en el que se ha visto afectado el derecho a la educación, identificar las consecuencias asociadas desde diferentes perspectivas y poder plantear propuestas y estrategias de reconstrucción para la que se conoce como nueva normalidad (Luengo y Manso, 2020). En cualquier caso, como establece Fernández-Enguita (2020), lo que ha puesto de manifiesto este confinamiento es la existencia de tres brechas en el sistema educativo español: la brecha de acceso (tener o no tener acceso a conexión y dispositivos tecnológicos); la brecha de uso (tiempo de uso y calidad del mismo); y la brecha escolar (habilidades del profesorado, 
disponibilidad). En este sentido, Luengo y Manso (2020, p. 13) establecen que 'existe una brecha de capital sociocultural en nuestra sociedad que se transfiere a la esfera de lo escolar' y que, aunque todos los sistemas educativos lo intentan mitigar en mayor o menor medida, la crisis actual parece amenazar con ampliar esta brecha escolar y curricular de manera significativa requiriendo ajustes a nivel de currículo, evaluación, programas, recursos y medidas sanitarias (Zubillaga y Gortazar, 2020). Según Jorquera (2020), en su informe de Save the Children, en España hay 2,1 millones de menores que viven en situación de pobreza y exclusión social, cuyas familias no se encuentran en situación de ayudarles en las labores escolares cuando desde los centros se reclama su participación (Martín-Criado y Gómez-Bueno, 2017). En el contexto actual, y ante una situación sin precedentes, los centros y los docentes han improvisado con los medios que tenían a su disposición una estructura que parece haber permitido a una buena parte de los estudiantes seguir el aprendizaje, aunque no a todo el alumnado (Díez-Gutiérrez y Gajardo-Espinoza, 2020b).

Respecto a la actuación de las familias, ésta ha sido muy diferente y desigual en función de su situación y contexto. Algunas han tenido la capacidad para atender a sus hijos, mientras que, en otros casos, ha sido verdaderamente complejo. Las principales razones san sido; a) consecuencia del teletrabajo coincidente con el periodo escolar; b) incapacidad para proporcionar a sus hijos la ayuda curricular que requerían (Beltrán, 2013; CifuentesFaura, 2020); c) falta de medios tecnológicos o de uso (Alva de la Selva, 2015; Banco Mundial, 2020a, 2020b); y d) por la falta de comunicación familia-escuela (Razeto, 2016). Son las familias más desfavorecidas social y económicamente las que han tenido más problemas para realizar el seguimiento.

En este sentido, es conocido el hecho de que los periodos de ausencia en los centros educativos, como ocurre en los periodos vacacionales, tienen una serie de consecuencias de desaprendizaje (Cooper et al. 1996; Fairchild y Boulay, 2003; Kerry y Davies, 1998; Shinwell y Defeyter, 2017). De hecho, las pérdidas de aprendizaje durante las vacaciones de verano hacen que la brecha en el rendimiento académico aumente (Borman, Benson y Overman, 2005; Patton y Reschly, 2013). Esta situación se agrava especialmente con el alumnado de necesidades educativas especiales o de contextos socioeconómicos desfavorecidos (Save the Children, 2020). En esta situación de confinamiento, las tareas demandadas para casa parecen haber potenciado aún más las desigualdades. Este aspecto, que ya identificaba Meirieu (2005), lo corroboran Posada y Taborda (2012) cuando demuestran que quienes tienen un entorno familiar y social empobrecido están en desventaja sobre aquellos que lo tienen enriquecido. Los estudiantes con rendimiento bajo están menos motivados intrínsecamente, tienen menos interés en hacer los deberes y requieren de mucha motivación extrínsecamente para realizarlo (Pan et al., 2013). Además, no se puede olvidar que hay una relación positiva entre el tiempo empleado en la realización de los deberes y el rendimiento académico en los últimos cursos de Secundaria y Bachillerato (Rosário et al., 2006). En cualquier caso, parece evidente que la capacidad para autorregularse es imprescindible para obtener un buen rendimiento académico (Fernández-Alonso, Suárez-Álvarez y Muñiz, 2014; Xu, 2010). y, por el contrario, los estudiantes que reciben más ayuda, también emplean más tiempo y obtienen peores resultados (Trautwein y Lüdtke, 2007), como ya se observó en España en algunas comunidades autónomas (Gobierno de Aragón, 2010; Gobierno Vasco, 2012).

Además de las medidas para resolver la brecha digital, para solucionar la brecha curricular es imprescindible atender a los modelos de enseñanza empleados por los docentes 
(Moreno y Luengo, 2020). En este sentido, las diferencias entre los docentes que siguen un modelo tradicional centrado en el libro de texto y los seguidores de un modelo competencial condicionan la posibilidad de salvar la brecha curricular. No se debe olvidar que los primeros utilizan métodos, técnicas y estrategias que centran su atención en lo meramente conceptual (Toro y Arguis, 2015), mientras que los segundos fomentan la motivación hacia el aprendizaje, su participación activa en las tareas, la colaboración entre el alumnado y el fomento de su autonomía y autorregulación (Casado, 2018; Zimmerman y Shunk, 2011). Es fundamental, y más en situación de confinamiento, que los docentes empleen metodologías activas que impliquen al alumnado en sus tareas, evitando así su desconexión y falta de seguimiento (Zubillaga y Gortazar, 2020); aunque no olvidando las distracciones generadas por la utilización del mismo ordenador o tablet para la adquisición del aprendizaje y su uso como herramienta de juego y disfrute (Bringa, Rodríguez y Herreroz, 2008; Fernández-Planells y Figuera, 2012).

La OCDE (2020) establece una guía para esta situación tan particular, fomentando la relación entre docentes, entre los centros y de estos con las familias. En este sentido, tanto Education Endowment Foundation (EEF, 2020), la UNESCO (2020) como Save the Children (2020) identifican una serie de factores a solventar para afrontar las situaciones de desigualdad en contextos desfavorecidos que deben ser completados y que son los siguientes: a) la calidad de la docencia asociada, b) asegurarse del acceso a la tecnología y su utilización limitando el número de plataformas, c) elegir metodologías adecuadas y activas, d) potenciar el trabajo colaborativo y cooperativo, e) desarrollar procesos de evaluación formativa con procedimientos de autoevaluación y coevaluación asociados a instrumentos claros y objetivos, f) desarrollar reglas de aprendizaje a distancia y controlar el proceso de aprendizaje de los estudiantes sin sobrecargas a las familias, y g) controlar los tiempos de atención en sesiones en vivo y fomentar el trabajo individual y autorregulados con planificadores. Sin embargo, todos estos factores de éxito que se plantean requieren partir de una serie de condiciones que es necesario valorar y que, en caso de no cumplirse, se estaría desaprovechando esta situación como elemento acelerador de la transformación social (Raya, 2020), así como la posibilidad de generar un verdadero cambio en el modelo educativo de nuestro país y que tanto necesita.

Este artículo plantea la necesidad de analizar los efectos del confinamiento en el proceso de aprendizaje de los estudiantes desde un posicionamiento holístico, procurando aprehender la realidad desde diferentes ópticas. Por esta razón, se ha dado voz a dos de los colectivos implicados en el aprendizaje de los estudiantes durante este periodo, las familias y los docentes. Cada uno de estos colectivos ha participado desde espacios distintos, aportando información con diferentes matices que permitirá describir de forma más completa y compleja la realidad. Así, se plantean dos objetivos a) analizar las variables relativas a la relación de la familia con los centros educativos y los docentes, así como sus problemas para apoyar a los alumnos; b) analizar el efecto que tiene el modelo de enseñanza del docente en el seguimiento de la enseñanza virtual, su formación en este tipo de enseñanza y el tiempo dedicado a las diferentes tareas docentes durante el confinamiento. Esto supone un aporte significativo a la literatura existente sobre la temática, ya que hasta el momento no se ha publicado ningún estudio en el ámbito español acerca del Covid 19 que utilice una muestra tan elevada de familias y docentes. 


\section{Método}

\section{Participantes}

Han participado un total de 9567 sujetos de los cuales 3700 son docentes y 5867 familias. En el Cuadro 1 se recogen las principales características de los participantes. En relación con los docentes, se observa que una amplia mayoría de ellos son mujeres, con más de 10 años de experiencia y trabajando en centro de titularidad pública. Respecto a las familias, la mitad de aquellas que ayudaban a los hijos en las tareas escolares tienen estudios universitarios y también la mitad no han teletrabajado durante el confinamiento. Para la selección de esta muestra se ha utilizado un procedimiento no aleatorio, intencional, utilizando como criterios de inclusión tener hijos en edad escolar (para el cuestionario a las familias) y ser docente de cualquier etapa (en el caso del cuestionario a los profesores). El acceso a los participantes se ha llevado a cabo mediante la técnica bola de nieve (snowball) que consiste en solicitar la participación a determinado número de personas accesibles que, a su vez, se lo hace llegar a otras y así sucesivamente hasta tener la muestra final (Ruiz, 2008).

Cuadro 1. Descripción de los participantes

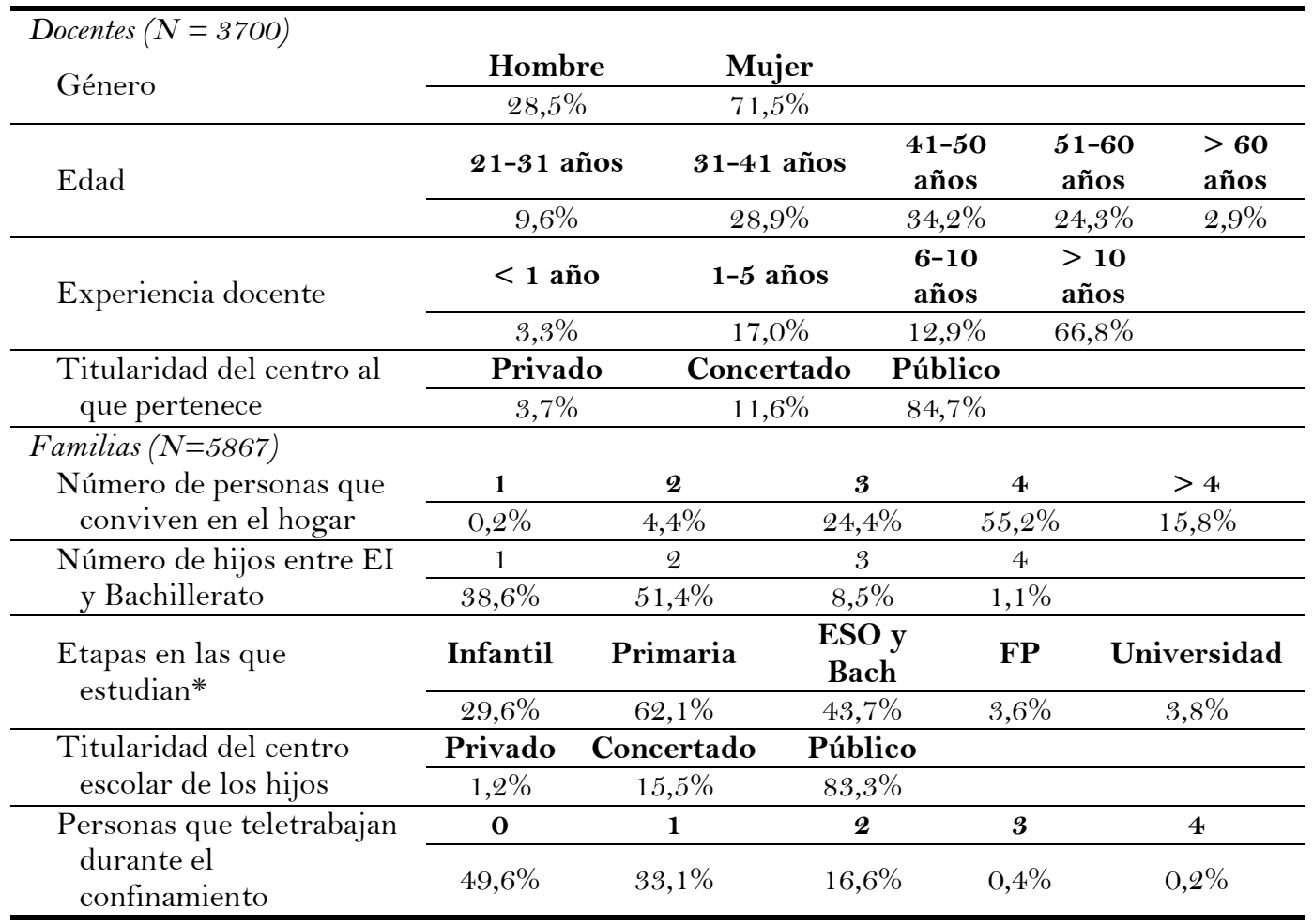

Nota: * Pregunta de respuesta múltiple, el total de las respuestas supera el 100\%

\section{Instrumentos}

Para la recogida de información se han utilizado dos cuestionarios (uno de familias y otro de docentes), elaborados expresamente para esta investigación. Como ha sido descrito anteriormente, el planteamiento de partida es describir el efecto del confinamiento de forma holística, por lo que se consideró necesario aportar la visión tanto de las familias como de los docentes para conseguir una visión lo más completa posible de la situación. La elaboración de los cuestionarios ha llevado asociado un proceso de validación de contenido por parte de seis expertos que ha consistido en el establecimiento de una 
estructura base desde la que invitar a participantes colaboradores en todo el proceso, y en la definición de evidencias que permitieran establecer hasta qué punto los ítems del cuestionario eran representativos de las conductas que a la vez se pretendían observar a partir del constructo teórico (López-López e Hinojosa, 2016). En base a las sugerencias recibidas se desarrollaron las versiones finales que han contado con un total de 48 ítems en el cuestionario de docentes y 26 en el de familias. Este proceso utilizado garantiza la validez de contenido, ya que cada experto valoró la objetividad, utilidad y pertinencia de cada pregunta a partir de los objetivos de la investigación, realizándose varias rondas hasta encontrar la mayor afinidad y coherencia. Los expertos fueron seleccionados en función de su experiencia docente y perfil investigador, siendo todos ellos profesionales con más de 15 años de experiencia y al menos cinco publicaciones de impacto en relación a la educación y el sistema educativo. Dado la naturaleza de la investigación y la tipología de las preguntas, se optó por esta vía de validación como las más óptima, descartando otros procesos de carácter más estadístico como análisis factoriales y pruebas psicométricas. Ambos cuestionarios tienen una misma estructura con una escala de respuesta de 4 categorías, donde 1 nulo/muy mal es y 4 es alto/muy bien, que incluye unas preguntas iniciales de tipo sociodemográfico, una segunda parte dedicada a las dificultades y respuesta dadas por distintos actores de la comunidad educativa y un bloque de preguntas sobre la intervención de los docentes y los modelos de enseñanza empleados.

\section{Diseño y procedimiento}

La investigación ha seguido un diseño ex post facto. Los cuestionarios han sido distribuidos por las redes sociales y contestados/cumplimentados a través de la herramienta formularios de Google entre los días 15 de abril y 8 de mayo del 2020. Antes de pasar a contestar el cuestionario se aseguraba el cumplimiento de todas las normas éticas requeridas tales como consentimiento informado escrito, derecho a la información, protección de datos personales, confidencialidad y anonimato, no-discriminación, gratuidad y opción de abandonar el estudio (McMillan y Schumacher, 2001).

Análisis de los datos

Para el primer objetivo de la investigación se han realizado análisis descriptivos con distribuciones de frecuencia relativa para cada una de las categorías de las variables. Para el segundo objetivo se han realizado análisis de varianza simple, ANOVA, para valorar si existen diferencias en las variables de interés en función del modelo de enseñanza en el que se sitúan los profesores. Se han aplicado las pruebas de normalidad y de homocedasticidad para comprobar que la muestra se ajusta a una distribución normal, pudiéndose aplicar la estadística inferencial paramétrica. Para establecer entre qué grupos se producen diferencias estadísticamente significativas se ha utilizado la prueba post hoc de comparación múltiple de Scheffe. Esta prueba compara las medias por pares de grupos utilizando como error típico la varianza intra-grupos. Utilizando el modelo de enseñanza como variable independiente se ha analizado su efecto sobre tres grupos de variables: variables relacionadas con las dificultades del alumnado para el seguimiento de la enseñanza virtual, variables relacionadas con la formación en enseñanza virtual del profesorado y variables relacionadas con el tiempo que ha dedicado el profesorado a las diferentes tareas docentes durante el confinamiento. El análisis de los datos se ha realizado con el programa IBM SPSS (versión 26). 


\section{Resultados}

Para responder a los dos objetivos del presente estudio, se presentan los principales hallazgos agrupados. En primer lugar, las variables relativas a la relación de la familia con los centros educativos y los docentes, así como sus problemas para apoyar a los alumnos. En segundo lugar, las relativas al modelo de enseñanza del docente en el seguimiento de la enseñanza virtual, su formación en este tipo de enseñanza y el tiempo dedicado a las diferentes tareas docentes durante el confinamiento.

El Cuadro 2 describe la percepción comparada de docentes y familias en relación a la forma en la que se ha producido la relación entre el centro y los docentes con las familias durante el periodo de confinamiento. Así, desde el punto de vista del profesorado, el conocimiento que tienen las familias sobre las claves del modelo de enseñanza virtual del centro es nulo $(14,8 \%)$ o escaso $(45,4 \%)$. Sin embargo, la mayoría de las familias consideran que la comunicación con el centro sobre el modelo a distancia durante el confinamiento ha sido buena $(50,6 \%)$ o muy buena $(14,2 \%)$, valorando la comunicación entre el profesorado y la familia como buena $(45,8 \%)$ o muy buena $(17,5 \%)$. Este resultado es apoyado por la mayoría de los docentes, que consideran como medio ( $47 \%)$ o muy alto $(18,7 \%)$ el apoyo de las familias a la actividad docente.

Cuadro 2. Relación de la familia con el centro y los docentes (porcentajes)

\begin{tabular}{lccccc}
\hline & POBLACIÓN & $\mathbf{1}$ & $\mathbf{2}$ & $\mathbf{3}$ & $\mathbf{4}$ \\
\hline $\begin{array}{c}\text { Conocimiento de las familias sobre el modelo } \\
\text { a distancia del centro }\end{array}$ & Docentes & $14,8 \%$ & $45,4 \%$ & $32,9 \%$ & $6,9 \%$ \\
\hline $\begin{array}{c}\text { Comunicación centro-familia sobre el modelo } \\
\text { a distancia }\end{array}$ & Familias & $9,0 \%$ & $26,2 \%$ & $50,6 \%$ & $14,2 \%$ \\
\hline $\begin{array}{c}\text { Comunicación profesorado y familia para } \\
\text { apoyar las tareas virtuales }\end{array}$ & Familias & $10,2 \%$ & $26,6 \%$ & $45,8 \%$ & $17,5 \%$ \\
\hline $\begin{array}{c}\text { Grado de satisfacción de las familias respecto } \\
\text { a la tutoría virtual }\end{array}$ & Profesores & $2,9 \%$ & $19,6 \%$ & $57,1 \%$ & $20,4 \%$ \\
\hline Apoyo de las familias a la actividad docente & Profesores & $6,3 \%$ & $28,1 \%$ & $47 \%$ & $18,7 \%$ \\
\hline
\end{tabular}

Nota: 1. Nulo/muy mal; 2. Bajo/regular; 3. Medio/bien; 4. Alto/muy bien.

Cuadro 3. Problemas de las familias para apoyar a los alumnos (porcentajes)

\begin{tabular}{|c|c|c|c|c|c|}
\hline & POBLACIÓN & 1 & 2 & 3 & 4 \\
\hline $\begin{array}{l}\text { Preparación previa del padre/madre para } \\
\text { ayudar al alumnado }\end{array}$ & Familias & $11,4 \%$ & $27,3 \%$ & $45,6 \%$ & $15,7 \%$ \\
\hline $\begin{array}{l}\text { Preparación previa de la familia para ayudar } \\
\text { al alumnado }\end{array}$ & Familias & $8,6 \%$ & $27,4 \%$ & $47,8 \%$ & $16,2 \%$ \\
\hline $\begin{array}{l}\text { Conocimientos de plataformas y } \\
\text { herramientas digitales }\end{array}$ & Familias & $10,4 \%$ & $30,2 \%$ & $43,8 \%$ & $15,6 \%$ \\
\hline $\begin{array}{l}\text { La Administración debe formar en } \\
\text { competencia digital a las familias }\end{array}$ & Familias & $7,0 \%$ & $19,5 \%$ & $53,5 \%$ & $20,0 \%$ \\
\hline $\begin{array}{l}\text { Ayuda de la Administración en materia de } \\
\text { formación digital para el profesorado }\end{array}$ & Profesorado & $28,9 \%$ & $46,8 \%$ & $21,2 \%$ & $3,0 \%$ \\
\hline $\begin{array}{l}\text { Capacidad para compaginar el teletrabajo } \\
\text { con la ayuda a las tareas escolares }\end{array}$ & Familias & $21,8 \%$ & $34,7 \%$ & $35,4 \%$ & $8,1 \%$ \\
\hline
\end{tabular}

Nota: 1. Nulo/muy mal; 2. Bajo/regular; 3. Medio/bien; 4. Alto/muy bien.

El Cuadro 3 describe la visión tanto de las familias como de los docentes en relación a algunos de los problemas que pueden haber surgido en este periodo. En este sentido, el $73,5 \%$ de las familias inciden en la importancia (media o alta) de que la administración las forme en competencia digital, algo compartido por los docentes que, en un $75,7 \%$, 
considera que la respuesta de la administración en materia de formación digital ha sido regular o mala. El $56,5 \%$ de las familias consideran mala o regular su capacidad para compaginar teletrabajo y ayudar a sus hijos con la tarea escolar. En menor medida, en torno a un $40 \%$ (valores 1 y 2 ) de las familias considera como una dificultad tanto su preparación previa como sus conocimientos de plataformas o herramientas digitales.

Para analizar el modelo de enseñanza en el que se sitúan los docentes se ha optado por recategorizar las respuestas al ítem 'Indique en la siguiente escala, en qué modelo de enseñanza se sitúa desde el 1: totalmente en un trabajo basado en contenidos y ejercicioshasta un 5: totalmente basado en elaboración propia de trabajos competenciales- formando los siguientes tres grupos:

- Modelo de enseñanza basado en contenidos (respuestas 1 y 2): compuesto por 311 sujetos $(8,4 \%)$.

- Modelo de enseñanza mixto (respuesta 3): compuesto por 1488 sujetos (40,2\%)

- Modelo de enseñanza basado en competencias (respuestas 4 y 5 ): compuesto por 1901 sujetos $(51,4 \%)$

La delimitación de estos modelos docentes atendió a la respuesta realizada por los participantes a tres preguntas relacionadas con las metodologías de enseñanza empleadas en el aula: a) nivel de importancia que deben de tener los contenidos en la enseñanza; b) nivel de importancia que debe de tener la metodología en la enseñanza; c) nivel de importancia que deben de tener la evaluación en la enseñanza. A partir de estas respuestas, se utilizó un sistema de ponderación y estratificación en tres niveles, asignando a los docentes con las puntuaciones más altas el Modelo de enseñanza basado en contenidos, las medias al Modelo de enseñanza mixto y las más bajas al Modelo de enseñanza basado en competencias. Tal como se observa en el Cuadro 4, el modelo de enseñanza en el que se sitúa el profesorado produce diferencias significativas en la importancia que los docentes dan a la falta de formación de los estudiantes, a la falta de recursos y a la falta de redes entre los estudiantes. De este modo, los profesores que se sitúan en un modelo mix to dan más peso a estas variables para explicar las dificultades en el seguimiento de la enseñanza virtual que los que se sitúan en un modelo basado en competencias.

En el Cuadro 5 se puede observar que, excepto en el caso de la formación oficial (ofrecida por la administración), se obtienen diferencias estadísticamente significativas tanto en los procesos de formación como en el nivel de competencia virtual del profesorado. Los profesores que se sitúan en un modelo basado en competencias tienen puntuaciones significativamente mayores en formación autodidacta $([\mathrm{df}=2,3697, \mathrm{~N}=3700] \mathrm{F}=$ $13,56, \mathrm{p}<0,01)$ y se sienten más competentes $([\mathrm{df}=2,3697, \mathrm{~N}=3700] \mathrm{F}=57,05, \mathrm{p}$ $<0,01)$ que los que se sitúan en modelos mixtos y basados en contenidos. 
Cuadro 4. Comparación de las medias en las razones que explican las dificultades en el seguimiento de la enseñanza virtual en función del modelo de enseñanza

\begin{tabular}{|c|c|c|c|c|c|c|}
\hline & & \multicolumn{4}{|c|}{ PRUEBA POST HOC (SCHEFFE) } & \multirow{2}{*}{ ANOVA } \\
\hline & & $\overline{\boldsymbol{X}}$ & $(1)$ & $(2)$ & $(3)$ & \\
\hline \multirow{6}{*}{$\begin{array}{l}\text { Falta de } \\
\text { formación }\end{array}$} & \multirow{2}{*}{ M. Cont. } & \multirow{2}{*}{2,50} & dif & & & \multirow{6}{*}{$\begin{array}{c}\operatorname{Df}(2,3697) \\
\mathrm{F}=9,81 \\
\mathrm{p}=0,000\end{array}$} \\
\hline & & & $\operatorname{sig}$ & & & \\
\hline & \multirow{2}{*}{ M. Mixto } & \multirow{2}{*}{2,57} & dif & & 0,140 & \\
\hline & & & $\operatorname{sig}$ & & 0,000 & \\
\hline & \multirow{2}{*}{ M. Comp. } & \multirow{2}{*}{2,43} & dif & $-0,140$ & & \\
\hline & & & $\operatorname{sig}$ & 0,000 & & \\
\hline \multirow{6}{*}{$\begin{array}{l}\text { Falta de } \\
\text { medios }\end{array}$} & \multirow{2}{*}{ M. Cont. } & \multirow{2}{*}{2,67} & dif & & & \multirow{6}{*}{$\begin{array}{c}\mathrm{Df}(2,3697) \\
\mathrm{F}=4,24 \\
\mathrm{p}=0,015\end{array}$} \\
\hline & & & $\operatorname{sig}$ & & & \\
\hline & \multirow{2}{*}{ M. Mixto } & \multirow{2}{*}{2,72} & dif & & 0,094 & \\
\hline & & & sig & & 0,015 & \\
\hline & \multirow{2}{*}{ M. Comp. } & \multirow{2}{*}{2,63} & dif & $-0,094$ & & \\
\hline & & & $\operatorname{sig}$ & 0,015 & & \\
\hline \multirow{3}{*}{$\begin{array}{l}\text { Falta de } \\
\text { apoyo de la } \\
\text { familia }\end{array}$} & M. Cont. & 2,39 & & & & \multirow{3}{*}{$\begin{array}{c}\mathrm{Df}(2,3697) \\
\mathrm{F}=1,62 \\
\mathrm{p}=0,198\end{array}$} \\
\hline & M. Mixto & 2,40 & & & & \\
\hline & M. Comp. & 2,34 & & & & \\
\hline \multirow{5}{*}{$\begin{array}{l}\text { Falta de redes } \\
\text { entre los } \\
\text { alumnos }\end{array}$} & \multirow{2}{*}{ M. Cont. } & \multirow{2}{*}{2,14} & dif & & & \multirow{5}{*}{$\begin{array}{c}\operatorname{Df}(2,3697) \\
\mathrm{F}=4,20 \\
\mathrm{p}=0,015\end{array}$} \\
\hline & & & $\operatorname{sig}$ & & & \\
\hline & \multirow{2}{*}{ M. Mixto } & \multirow{2}{*}{2,24} & dif & & 0,081 & \\
\hline & & & $\frac{\operatorname{sig}}{\operatorname{dif}}$ & & 0,025 & \\
\hline & M. Comp. & 2,16 & sig & 0,025 & & \\
\hline \multirow{3}{*}{$\begin{array}{l}\text { Falta } \\
\text { coordinación } \\
\text { profesores }\end{array}$} & M. Cont. & 1,76 & & & & \multirow{3}{*}{$\begin{array}{c}\operatorname{Df}(2,3697) \\
\mathrm{F}=0,51 \\
\mathrm{p}=0,599\end{array}$} \\
\hline & M. Mixto & 1,72 & & & & \\
\hline & M. Comp. & 1,71 & & & & \\
\hline & M. Cont. & 2,14 & & & & $\operatorname{Df}(2,3697)$ \\
\hline $\begin{array}{l}1 \text { area } \\
\text { excesiva }\end{array}$ & M. Mixto & 2,07 & & & & $\mathrm{~F}=1,70$ \\
\hline & M. Comp. & 2,12 & & & & $\mathrm{p}=0,182$ \\
\hline Falta & M. Cont. & 2,74 & & & & $\operatorname{Df}(2,3697)$ \\
\hline autorregulaci & M. Mixto & 2,67 & & & & $\mathrm{~F}=1,64$ \\
\hline ón & M. Comp. & 2,64 & & & & $\mathrm{p}=0,194$ \\
\hline
\end{tabular}

Nota: Sólo se muestras las diferencias entre medias significativas $(\mathrm{p}<0,01)$.

La formación en el centro es significativamente menos realizada por los profesores que se sitúan en un modelo basado en contenidos ( $[\mathrm{df}=2,3697, \mathrm{~N}=3700] \mathrm{F}=9,90, \mathrm{p}<0,01$ ) que los de los otros dos grupos. Por último, en la formación en metodología y evaluación virtual se establecen diferencias significativas entre los tres grupos $([\mathrm{df}=2,3697, \mathrm{~N}=$ $3700] \mathrm{F}=32,35, \mathrm{p}<0,01)$ siendo las mayores puntuaciones las de los profesores con enseñanza basada en competencias, intermedias las de los modelos mix tos y las más bajas asociadas a los docentes que se basan en contenidos.

Cuadro 5. Comparación de las medias en formación y nivel de competencia en función del modelo de enseñanza

\begin{tabular}{|c|c|c|c|c|c|c|}
\hline & & \multicolumn{4}{|c|}{ PRUEBA POST HOC (SCHEFFE) } & \multirow{2}{*}{ ANOVA } \\
\hline & & $\overline{\boldsymbol{X}}$ & $(1)$ & (2) & (3) & \\
\hline \multirow{3}{*}{$\begin{array}{l}\text { Formación } \\
\text { oficial }\end{array}$} & M. Cont. & 2,30 & & & & $\operatorname{Df}(2,3697)$ \\
\hline & M. Mixto & 2,39 & & & & $\mathrm{~F}=1,34$ \\
\hline & M. Comp. & 2,36 & & & & $\mathrm{p}=0,26$ \\
\hline \multirow{3}{*}{$\begin{array}{l}\text { Formación } \\
\text { autodidacta }\end{array}$} & \multirow{2}{*}{ M. Cont. } & \multirow{2}{*}{3,21} & dif & & $-0,182$ & $\operatorname{Df}(2,3697)$ \\
\hline & & & $\operatorname{sig}$ & & 0,000 & $\mathrm{~F}=13,56$ \\
\hline & M. Mixto & 3,29 & dif & & $-0,99$ & $\mathrm{p}=0,00$ \\
\hline
\end{tabular}




\begin{tabular}{|c|c|c|c|c|c|c|c|}
\hline & & & $\operatorname{sig}$ & & & 0,000 & \\
\hline & M Comn & 800 & dif & 0,182 & 0,099 & & \\
\hline & M. Comp. & 3,39 & sig & 0,000 & 0,000 & & \\
\hline \multirow{6}{*}{$\begin{array}{l}\text { Formación en } \\
\text { el centro }\end{array}$} & \multirow{2}{*}{ M. Cont. } & \multirow{2}{*}{2,11} & dif & & $-0,226$ & $-0,268$ & \multirow{6}{*}{$\begin{array}{c}\operatorname{Df}(2,3697) \\
\mathrm{F}=9,90 \\
\mathrm{p}=0,00\end{array}$} \\
\hline & & & sig & & 0,001 & 0,000 & \\
\hline & \multirow{2}{*}{ M. Mixto } & \multirow{2}{*}{2,33} & dif & 0,226 & & & \\
\hline & & & $\operatorname{sig}$ & 0,000 & & & \\
\hline & \multirow{2}{*}{ M. Comp. } & \multirow{2}{*}{2,37} & dif & 0,268 & & & \\
\hline & & & sig & 0,000 & & & \\
\hline \multirow{6}{*}{$\begin{array}{l}\text { Formación en } \\
\text { metodologías } \\
\text { y evaluación } \\
\text { virtual }\end{array}$} & \multirow{2}{*}{ M. Cont. } & \multirow{2}{*}{1,92} & dif & & $-0,282$ & $-0,443$ & \multirow{6}{*}{$\begin{array}{c}\operatorname{Df}(2,3697) \\
\mathrm{F}=32,35 \\
\mathrm{p}=0,00\end{array}$} \\
\hline & & & $\operatorname{sig}$ & & 0,000 & 0,000 & \\
\hline & \multirow{2}{*}{ M. Mixto } & \multirow{2}{*}{2,2} & dif & 0,282 & & $-0,161$ & \\
\hline & & & $\operatorname{sig}$ & 0,000 & & 0,000 & \\
\hline & \multirow{2}{*}{ M. Comp. } & \multirow{2}{*}{2,36} & dif & 0,443 & 0,161 & & \\
\hline & & & sig & 0,000 & 0,000 & & \\
\hline \multirow{6}{*}{$\begin{array}{l}\text { Nivel de } \\
\text { competencia } \\
\text { virtual }\end{array}$} & \multirow{2}{*}{ M. Cont. } & \multirow{2}{*}{2,54} & dif & & $-0,173$ & $-0,416$ & \multirow{6}{*}{$\begin{array}{c}\mathrm{Df}(2,3697) \\
\mathrm{F}=57.05 \\
\mathrm{p}=0,00\end{array}$} \\
\hline & & & $\operatorname{sig}$ & & 0,003 & 0,000 & \\
\hline & \multirow{2}{*}{ M. Mixto } & \multirow{2}{*}{2,72} & dif & 0,173 & & $-0,243$ & \\
\hline & & & sig & 0,000 & & 0,000 & \\
\hline & \multirow{2}{*}{ M. Comp. } & \multirow{2}{*}{2,96} & dif & 0,416 & 0,243 & & \\
\hline & & & $\operatorname{sig}$ & 0,000 & 0,000 & & \\
\hline
\end{tabular}

Nota: Sólo se muestras las diferencias entre medias significativas $(\mathrm{p}<0,01)$.

Por último, el Cuadro 6 describe las diferencias en los tiempos que el profesorado dedica a las tareas en función del modelo en el que se sitúan. Como se observa, los profesores que se sitúan en un modelo competencial dedican un porcentaje mayor de tiempo a todas las tareas docentes excepto a la corrección de tareas. Esta situación se invierte exactamente para los profesores con un perfil basado en contenidos, que dedican menos tiempo a todas las tareas excepto a la corrección. Los profesores del modelo mixto alcanzan puntuaciones intermedias excepto en la corrección de tareas, en la que alcanzan la mayor puntuación de los tres grupos. La prueba de comparación múltiple de Scheffe señala que las diferencias son significativas entre los profesores del modelo competencial y del modelo basado en contenidos en el tiempo dedicado (mayor en todos los casos para los primeros) a resolver dudas $\left(\bar{X}_{\mathrm{a}-} \bar{X}_{\mathrm{b}}=3,67, \mathrm{p}<0,01\right)$ y evaluar aprendizaje $\left(\bar{X}_{\mathrm{a}} \bar{X}_{\mathrm{b}}=3,11, \mathrm{p}<0,05\right)$. Las diferencias con los del modelo mixto se establecen en el tiempo dedicado a preparar clases, mayor en los del modelo basado en competencias $\left(\bar{X}_{\mathrm{a}} \bar{X}_{\mathrm{b}}=2,14, \mathrm{p}<0,05\right)$ y a corregir tareas, mayor para los del modelo mixto $\left(\bar{X}_{\mathrm{a}} \bar{X}_{\mathrm{b}}=2,7 \mathrm{O}, \mathrm{p}<0,01\right)$.

Cuadro 6. Comparación de las medias en tiempo dedicado a las tareas en función del modelo de enseñanza

\begin{tabular}{|c|c|c|c|c|c|c|c|}
\hline & \multicolumn{6}{|c|}{ PRUEBA POST HOC (SCHEFFE) } & \multirow{2}{*}{ ANOVA } \\
\hline & & $\overline{\boldsymbol{X}}$ & & $(1)$ & $(2)$ & $(3)$ & \\
\hline \multirow{6}{*}{$\begin{array}{l}\text { Preparar } \\
\text { clases }\end{array}$} & \multirow{2}{*}{ M. Cont. } & \multirow{2}{*}{37,65} & dif & & & & \multirow{6}{*}{$\begin{array}{l}\mathrm{Df}(2,3697) \\
\mathrm{F}=5,42 \\
\mathrm{p}=0,004\end{array}$} \\
\hline & & & $\operatorname{sig}$ & & & & \\
\hline & \multirow{2}{*}{ M. Mixto } & \multirow{2}{*}{38,33} & dif & & & $-2,14$ & \\
\hline & & & sig & & & 0,014 & \\
\hline & \multirow{2}{*}{ M. Comp. } & \multirow{2}{*}{40,47} & dif & & 2,14 & & \\
\hline & & & $\operatorname{sig}$ & & 0,014 & & \\
\hline \multirow{5}{*}{$\begin{array}{l}\text { Resolver } \\
\text { dudas }\end{array}$} & \multirow{2}{*}{ M. Cont. } & \multirow{2}{*}{23,57} & dif & & & $-3,674$ & \multirow{5}{*}{$\begin{array}{l}\operatorname{Df}(2,3697) \\
F=5,12 \\
p=0,006\end{array}$} \\
\hline & & & $\operatorname{sig}$ & & & 0,009 & \\
\hline & \multirow{2}{*}{ M. Mixto } & \multirow{2}{*}{26,16} & dif & & & & \\
\hline & & & $\operatorname{sig}$ & & & & \\
\hline & M. Comp. & 27,24 & dif & 3,67 & & & \\
\hline
\end{tabular}




\begin{tabular}{|c|c|c|c|c|c|c|c|}
\hline & & & sig & 0,009 & & & \\
\hline \multirow{6}{*}{$\begin{array}{l}\text { Corregir } \\
\text { tareas }\end{array}$} & \multirow{2}{*}{ M. Cont. } & \multirow{2}{*}{30,39} & dif & & & & \multirow{6}{*}{$\begin{array}{l}\text { Df }(2,3697) \\
\mathrm{F}=7,62 \\
\mathrm{p}=0,00\end{array}$} \\
\hline & & & sig & & & & \\
\hline & \multirow{2}{*}{ M. Mixto } & \multirow{2}{*}{30,52} & dif & & & 2,695 & \\
\hline & & & $\operatorname{sig}$ & & & 0,001 & \\
\hline & \multirow{2}{*}{ M. Comp. } & \multirow{2}{*}{27,82} & dif & & $-2,695$ & & \\
\hline & & & $\operatorname{sig}$ & & 0,001 & & \\
\hline \multirow{6}{*}{$\begin{array}{l}\text { Evaluar } \\
\text { aprendizajes }\end{array}$} & \multirow{2}{*}{ M. Cont. } & \multirow{2}{*}{19,26} & dif & & & -3.107 & \multirow{6}{*}{$\begin{array}{l}\mathrm{Df}(2,3697) \\
\mathrm{F}=3,51 \mathrm{p}= \\
\text { 0,030 }\end{array}$} \\
\hline & & & $\operatorname{sig}$ & & & 0,042 & \\
\hline & \multirow{2}{*}{ M. Mixto } & \multirow{2}{*}{21,38} & dif & & & & \\
\hline & & & $\operatorname{sig}$ & & & & \\
\hline & \multirow{2}{*}{ M. Comp. } & \multirow{2}{*}{22,37} & dif & 3,107 & & & \\
\hline & & & $\operatorname{sig}$ & 0,042 & & & \\
\hline
\end{tabular}

Nota: Sólo se muestras las diferencias entre medias significativas $(\mathrm{p}<0,01)$.

\section{Discusión}

Los objetivos del presente estudio fueron dos; a) analizar las variables relativas a la relación de la familia con los centros educativos y los docentes, así como sus problemas para apoyar a los alumnos; b) analizar el efecto que tiene el modelo de enseñanza del docente en el seguimiento de la enseñanza virtual, su formación en este tipo de enseñanza y el tiempo dedicado a las diferentes tareas docentes durante el confinamiento.

En relación al primer objetivo, se ha mostrado el escaso conocimiento que han manifestado las familias en relación a la enseñanza virtual. Esto demuestra que, a pesar de la gran inmersión tecnológica que ha experimentado la sociedad en la última década, esta no se asocia a fines pedagógicos y de enseñanza. Para ello, es necesario que se realicen cambios políticos y educativos inmediatos (Nepo, 2017). El uso de la tecnología está más vinculada al ocio y al divertimento que a cualquier otro ámbito, lo que provoca que la efectividad del proceso de enseñanza no se haya visto mejorada sustancialmente como podría preverse. Lo más relevante no es la tecnología en sí misma, sino el uso que se haga de la misma, siendo en todo caso fundamental la labor del docente (Oblinger, 2018). Los datos del estudio han reflejado cómo las familias valoran positivamente la comunicación mantenida tanto con el centro escolar como con el profesorado. Esto es recíproco, ya que los docentes consideran adecuado el apoyo de las familias. Es un dato de verdadero interés en el ámbito educativo, ya que la comunicación bidireccional entre el centro y las familias es uno de los factores que más incide en el clima motivacional y el rendimiento académico del alumnado (Sadiku y Sylaj, 2019). Sin embargo, los resultados demuestran cómo tanto el profesorado como las familias consideran que la respuesta de la administración no ha sido positiva, indicando que les tendría que haber formado en competencia digital. El rol de la administración suele ser muy cuestionado en el ámbito educativo, más si cabe en situaciones excepcionales que generan tantos cambios como la del confinamiento. Es fundamental que tenga unos criterios sólidos y claros de actuación que permitan ser aplicados de forma eficiente por encima de cualquier ideología política, algo que no siempre sucede (Jiménez-Eguizábal, Palmero y Luis-Rico, 2013). Esta idea cobra todavía más sentido al observar en los resultados del presente estudio la elevada disparidad existente en la tipología de familias y su capacidad y posibilidades para compaginar las tareas escolares y el teletrabajo.

En relación al segundo objetivo del estudio, se ha observado cómo el modelo de enseñanza utilizado por el profesor (competencial, mixto o basado en contenidos) influye 
significativamente en las variables relacionadas con la formación de los estudiantes y la falta de recursos, siendo los que utilizan un enfoque competencial los que perciben menos dificultades en el seguimiento de la enseñanza virtual. Es de elevada trascendencia que la docencia se entienda como algo que va más allá del profesor frente a los alumnos, normalmente con un libro de texto, dando información. El aprendizaje ha de ser 'ubicuo', permitiendo su transferibilidad a cualquier espacio, contexto y tiempo. Por otro lado, es fundamental trabajar intencionadamente en el aula la autonomía de los estudiantes, con el fin de que adquieran recursos de actuación que reduzcan la excesiva dependencia del docente y deriven un mayor compromiso hacia el aprendizaje (Näkk y Timoštšuk, 2019). Esta les permitirá adquirir una mayor capacidad de autorregulación de las tareas, utilizando para ello diferentes canales y fuentes de información con el fin de reducir la postergación y la falta de implicación (Iwamoto et al., 2017). De este modo, afrontar cualquier tipo de enseñanza online será más factible y motivante.

Los resultados del estudio han reflejado diferencias significativas en los diferentes tipos de formación y en su nivel de competencia percibida por parte del profesorado. Los que utilizan un modelo competencial son más autodidactas y se sienten más competentes que los que se sitúan en modelos mixtos y basados en contenidos. Es precisamente la enseñanza basada en competencias la que ha demostrado una mayor flexibilidad en las estrategias de enseñanza, tratando de conexionar el ámbito específico del conocimiento disciplinar con el desarrollo de habilidades transferibles a diversidad de contextos (Gulikers, Runhaar y Mulder, 2018). Esto implica que la información ha de provenir de diferentes fuentes, debiendo de ser comprendida (no solamente conocida) para poder interrelacionarla tanto con conocimientos previos como entre los propios estudiantes (Kim et al., 2016). Cuando los docentes conciben exclusivamente la enseñanza en la impartición de determinados contenidos para su posterior memorización por parte del alumnado, suelen encontrar mayores problemas y limitaciones para hacer la transición hacia metodologías más activas y participativas que requieran otro tipo de recursos (Tularam y Machisella, 2018).

En relación al tiempo invertido en la elaboración de las tareas, los resultados del estudio muestran cómo los docentes que enfocan la enseñanza bajo un modelo competencial dedican más tiempo a la elaboración de las tareas, a la preparación de las clases y a la resolución de dudas que el resto de docentes. Esto denota una mayor implicación del docente, que, aun asumiendo que la carga de trabajo sea mayor que al utilizar enfoques más tradicionales, decide aplicarlos por el mayor aprendizaje que supone para el alumnado (Vogelzang, Admiraal y Van Driel, 2020). Se demuestra cómo en esta situación de confinamiento los docentes con enfoques más basados en el contenido han seguido manteniendo la idea unidireccional de mandar tareas al alumnado o colgar documentos en plataforma, en lugar de repensar el planteamiento hacia otro tipo de actividades más significativas para los estudiantes. Es precisamente la variedad en la tipología de tareas y el feedback existente entre estudiante y docente a lo largo del proceso lo que genera un verdadero aprendizaje significativo (Dann, 2019). Los resultados también reflejan que los docentes competenciales dedican más tiempo a la evaluación del aprendizaje, pero no así a la corrección donde los docentes del enfoque de contenidos dedican más tiempo. Esto refleja la confusión existente entre evaluar y calificar, considerándolos términos equivalentes cuando difieren sustancialmente. Si bien la calificación se refiere a la nota numérica asignada a la finalización de un proceso, la evaluación debe de ser sinónimo de aprendizaje, quedando estrechamente relacionada la evaluación formativa y compartida 
con la adquisición de competencias específicas y transversales (Hortigüela, Palacios y López-Pastor, 2019).

\section{Conclusiones}

El periodo de confinamiento ha dejado tras de sí diversidad de secuelas educativas. Las familias se han visto claramente limitadas para poder atender con garantías a las tareas virtuales, aunque reconocen que la comunicación con el centro y los docentes ha sido positiva. Es la administración la peor parada, ya que tanto los docentes como las familias consideran que no ha cumplido con eficacia su función. Ha quedado de manifiesto la necesidad de trabajar la autonomía y la autorregulación de los estudiantes, siendo los enfoques competenciales empleados por los docentes los que han percibido menos limitaciones con la enseñanza virtual y han dedicado mayor tiempo a la preparación de clases y a la evaluación de las tareas.

Los resultados de la presente investigación suponen un aporte significativo a la situación de confinamiento educativo vivida en España, ya que en ningún estudio previo se había utilizado un número de familias y docentes tan elevado. Sin embargo, el estudio presenta algunas limitaciones. En primer lugar, no se clasifica a las familias en función de su nivel socioeconómico. En segundo lugar, no se atiende a la percepción de los estudiantes. Como futuras líneas de investigación sería pertinente poder arrojar datos cualitativos que den voz a las familias más desfavorecidas, así como comprobar cómo perciben los alumnos el aprendizaje adquirido en función de los enfoques metodológicos empleados por los docentes. Consideramos este artículo de especial interés para todas las administraciones educativas, ya que les permitirá conocer los datos reales sobre los que estructurar sus directrices educativas. También para los centros educativos y sus respectivos docentes, ya que, además de fomentar la reflexión sobre lo acontecido, podrá favorecer el establecimiento de protocolos de actuación ante un futuro rebrote. Es fundamental seguir investigando acerca de una situación inédita a nivel mundial, que, sin duda, ha de ser entendida como una oportunidad para reconducir determinados planteamientos y enfoques educativos.

\section{Referencias}

Alva de la Selva, A. R. (2015). Los nuevos rostros de la desigualdad en el siglo XXI: La brecha digital. Revista Mexicana de Ciencias Políticas y Sociales, 60(223), 265-285.

https://doi.org/10.1016/So185-1918(15)72138-O

Banco Mundial. (2020a). Education response note to Covid-19 in Europe and Central Asia. Banco Mundial.

Banco Mundial. (2020b). Remote learning, distance education and online learning during the Covid19 pandemic. Banco Mundial.

Beltrán, A. (2013). El tiempo de la familia es un recurso escaso: ¿Cómo afecta su distribución en el desempeño escolar? Apuntes: Revista de Ciencias Sociales, 4O(72), 117-156. https://doi.org/10.21678/apuntes.72.677

Borman, G. D., Benson, J. y Overman, L. T. (2005). Families, schools, and summer learning. The Elementary School Journal, 106(2), 131-150. https://doi.org/10.1086/499195 
Bringa, C., Rodríguez, F. J. y Herreroz, F. J. (2008). Adaptación y motivación escolar: Análisis de la influencia del consumo de medios electrónicos de comunicación por adolescentes. Cuadernos de Trabajo Social, 21, 141-143.

Casado, O. M. (2018). La autorregulación en el aula de educación primaria: Estudio y aplicación de un modelo integral de transición activa hacia la autonomía (Tesis doctoral). Universidad de Valladolid, España.

Cifuentes-Faura, J. (2020). Consecuencias en los niños del cierre de escuelas por Covid-19: El papel del gobierno, profesores y padres. Revista Internacional de Educación para la Justicia Social, $9(3)$.

Cooper, H., Nye, B., Charlton, K., Lindsay, J. y Greathouse, S. (1996). The effects of summer vacation on achievement test scores: A narrative and meta-analytic review. Review of Educational Research, 66(3), 227-268. https://doi.org/10.3102/00346543066003227

Constitución Española. (1978). Constitución española. https://www.boe.es/buscar/act.php?id=BOE-A-1978-31229

Dann, R. (2019). Feedback as a relational concept in the classroom. Curriculum Journal, 30(4), 352374. https://doi.org/10.1080/09585176.2019.1636839

Díez-Gutiérrez, E. J. y Gajardo-Espinoza, K. (2020a). Políticas educativas en tiempos de coronavirus: La confrontación ideológica en España. Revista Internacional de Educación para la Justicia Social, 2020, 9(3), 83-101. https://doi.org/10.15366/riejs2020.9.3.005

Díez-Gutiérrez, E. J. y Gajardo-Espinoza, K. (2020b). Educar y evaluar en tiempos de coronavirus: La situación en España. Multidisciplinary Journal of Educational Research, 10(2), 102-134. https://doi.org/10.447/remie.2020.5604

Education Endowment Foundation-EEF. (2020). Impact of school closures on the attainment gap: Rapid evidence assessment. Education Endowment Foundation.

Fairchild, R. y Boulay, M. (2003). Summer learning loss: The evidence and a possible solution. APPAM.

Fernández-Alonso, R., Suárez-Álvarez, J. y Muñiz, J. (2014). Tareas escolares en el hogar y rendimiento en matemáticas: Una aproximación multinivel con estudiantes de enseñanza primaria. Revista de Psicología y Educación, 9(2), 23-41.

Fernández-Enguita, M. (31 de marzo de 2020). Una pandemia imprevisible ha traído la brecha previsible. Cuaderno de Campo. https://blog.enguita.info/

Fernández-Franco, F. J. (2020). Estudio comparado de las normas del tercer trimestre del curso 2019/2020 reguladas por las administraciones educativas. USIE.

Fernández-Planells, A. y Figuera, M. (2012). Internet en las tareas escolares ¿iobstáculo u oportunidad? El impacto de la red en los hábitos de estudio de alumnos de secundaria de Barcelona y Lima. Sphera Publica, 12, 161-182.

Fernández-Riquelme, S. (2020). Primera historia de la crisis del coronavirus en España. La Razón Histórica, 46, 12-22.

Gobierno de Aragón. (2010). Evaluación de diagnóstico en Aragón 2010. Departamento de Educación, Cultura y Deporte.

Gobierno Vasco. (2012). Evaluación diagnóstica. Informe de resultados y análisis de variables $2^{\circ}$ educación secundaria obligatoria. Instituto Vasco de Evaluación e Investigación Educativa.

Gulikers, J., Runhaar, P. y Mulder, M. (2018). An assessment innovation as flywheel for changing teaching and learning. Journal of Vocational Education and Training, 70(2), 212-231.

https://doi.org/10.1080/13636820.2017.1394353 
Hortigüela, D., Palacios, A. y López-Pastor, V. (2019). The impact of formative and shared or coassessment on the acquisition of transversal competences in higher education. Assessment $y$ Evaluation in Higher Education, 44(6), 933-945. https://doi.org/10.1080/02602938.2018.1530341

Iwamoto, D. H., Hargis, J., Bordner, R. y Chandler, P. (2017). Self-regulated learning as a critical attribute for successful teaching and learning. International Journal for the Scholarship of Teaching and Learning, $11(2)$, 1-12. https://doi.org/10.20429/ijsotl.2017.110207

Jiménez-Eguizábal, A., Palmero, C. y Luis-Rico, I. (2013). The spectre of educational policy spreads throughout Europe. Its presence as a discipline in postgraduate education. Journal of New Approaches in Educational Research, 2(2), 82-87. https://doi.org/10.7821/naer.2.2.82-87

Jorquera, G. (2020). Familias en riesgo, análisis de la situación de pobreza en los hogares con hijos e hijas en España. Save the Children.

Kerry, T.y Davies, B. (1998). Summer learning loss: The evidence and a possible solution. Support for Learning, 13(3), 118-122. https://doi.org/10.1111/1467-9604.00072

Kim, S., Kalish, C. W., Weisman, K., Johnson, M. V. y Shutts, K. (2016). Young children choose to inform previously knowledgeable others. Journal of Cognition and Development, $17(2)$, 320340. https://doi.org/10.1080/15248372.2014.952731

López-López, M. C. e Hinojosa, E. F. (2016). Construction and validation of a questionnaire to study future teachers' beliefs about cultural diversity. International Journal of Inclusive Education, 20(5), 503-519. https://doi.org/10.1080/13603116.2015.1095249

Luengo, F. y Manso, J. (2020). Informe de investigación Covid19. Voces de docentes y familias. http://www.proyectoatlantida.eu/wordpress/informe-de-investigacion-covid 19

Martín-Criado, E. y Gómez-Bueno, C. (2017). El mito de la dimisión parental. Implicación familiar, desigualdad social y éxito escolar. Cuadernos de Relaciones Laborales, 35(2), 305-325. https://doi.org/10.5209/CRLA.56777

McMillan, J. H. y Schumacher, S. (2001). Investigación educativa: Una introducción conceptual. Pearson.

Meirieu, P. (2005). Los deberes en casa. Octaedro.

Moreno, J. M. y Luengo, F. (2020). Hay que parar la hemorragia educativa. https://www.magisnet.com/2020/06/hay-que-parar-la-hemorragia-educativa/

Näkk, A. M. y Timoštšuk, I. (2019). The dynamics of learning engagement and its relationship with teachers' classroom practices in primary school. Education, 47(1), 89-100. https://doi.org/10.1080/03004279.2017.1404620

Nepo, K. (2017). The use of technology to improve education. Child y Touth Care Forum, 46(2), 207221. https://doi.org/10.1007/s 10566-016-9386-6

Oblinger, D. G. (2018). It's not just about the technology. It's what you do with it that counts. Change: The Magazine of Higher Learning, 50(4), 40-43.

https://doi.org/10.1080/00091383.2018.1509584

OCDE. (2020). Learning remotely when schools close: How well are students and schools prepared? Insights from PISA. OCDE.

Pan, I., Regueiro, B., Ponte, B., Rodríguez, S., Piñeiro, I. y Valle, A. (2013). Motivación, implicación en los deberes escolares y rendimientos académico. Aula Abierta, 41(3), 13-22.

Patton, K. y Reschly, A. (2013). Using curriculum-based measurement to examine summer learning loss. Psychology in the Schools, 50(7), 738-753. https://doi.org/10.1002/pits.21704 
Posada, D. M. y Taborda, M. A. (2012). Reflexiones sobre la pertinencia de las tareas escolares: Acercamientos para futuros estudios. Uni-pluriversidad, 12(2), 22-33.

Raya, A. (6 de abril de 2020). Coronavirus, la disruption que acelera el cambio y la toma de conciencia. AndresRaya.com.

Razeto, A. (2016). El involucramiento de las familias en la educación de los niños: Cuatro reflexiones para fortalecer la relación entre familias y escuelas. Páginas de Educación, 9(2), 184-201. https://doi.org/10.22235/pe.v9i2.1298

Rosário, P., Mourão, R., Núñez, J.C., González-Pienda, J.A. y Solano, P. (2006). Escuela-familia: ¿Es posible una relación recíproca y positiva? Papeles del Psicólogo, 27(3), 174-182.

Ruiz, A. (2008) La muestra: Algunos elementos para su confección. Revista d'Innovació i Recerca en Educació, 1, 75-88. https://doi.org/10.1344/reire2008.1.1117

Sadiku, G. S. y Sylaj, V. (2019). Factors that influence the level of the academic performance of the students. Journal of Social Studies Education Research, 10(3),17-38.

Save the Children. (2020). Covid-19: Cerrar la brecha. Impacto educativo y propuestas de equidad para la desescalada. Save the Children.

Shinwell, J. y Defeyter, M. A. (2017). Investigation of summer learning loss in the UK. Front Public Health, 5, 1-7. https://doi.org/10.3389/fpubh.2017.00270

Trautwein, U. y Lüdtke, O. (2007). Esfuerzo y tiempo autoinformado por los alumnos en la tarea en seis asignaturas escolares: Diferencias entre alumnos y variaciones entre alumnos. Revista de Psicología Educativa, 99(2), 432-444.

Toro, A. y Arguis, M. (2015). Metodologías activas. A Tres Bandas, 38, 69-77.

Tularam, G. A. y Machisella, P. (2018). Traditional vs non-traditional teaching and learning strategies: The case of e-learning! International Journal for Mathematics Teaching and Learning, 19(1), 129-158.

UNESCO. (2020b). Covid-19: 10 Recommendations to plan distance learning solutions. UNESCO.

Vogelzang, J., Admiraal, W. F. y Van Driel, J. H. (2020). A teacher perspective on scrum methodology in secondary chemistry education. Chemistry Education Research and Practice, 21(1), 237-249. https://doi.org/10.1039/C9RPOO111E

$\mathrm{Xu}$, J. (2010). Predicting homework time management at the secondary school level: A multilevel analysis. Learning and individual differences, 20, 34-39.

https://doi.org/10.1016/j.lindif.2009.11.001

Zimmerman, B. J. y Shunk, D. H. (2011). Handbook of self-regulation of learning and performance Routledge.

Zubillaga, A. y Gortazar, L (2020). Covid-19 y educación: Problemas, respuestas y escenarios. Fundación Cotec para la Innovación.

\section{Breve CV de los/as autores/as}

\section{David Hortigüela-Alcalá}

Profesor Titular de Universidad. Director del Área de Didáctica de la Expresión Corporal. Secretario del Departamento de Didáctica Específicas. Autor de más de 250 publicaciones relacionadas con la innovación en educación, la educación física, la evaluación y el uso de los modelos pedagógicos. Revisor en más de 40 revistas científicas. Nombrado segundo 
mejor docente universitario de España en 2018 (premios Abanca). Estancias de investigación en diversidad de países. Director del grupo de investigación Enseñanza e Investigación en Educación Física (ENIEF) y del de innovación Pedagogía de la Educación Física y el Deporte (PEFIDE). Perfil de investigación: http://bit.ly/dhortigüelacv. ORCID ID: https://orcid.org/0000-0001-5951-758X. Email: dhortiguela@ubu.es

\section{Ángel Pérez-Pueyo}

Profesor Titular de Universidad. Director del Área de Extensión Universitaria. Director del Grupo Internivelar e Interdisciplinar Actitudes. Experto en competencias, evaluación y enseñanza de la Educación Física. Coordinador del Máster de Educación Secundaria en Universidad de León. Autor de diversidad de artículos y ponente en múltiples eventos, congresos y seminarios relacionados con el ámbito educativo. Integrante de varios grupos de investigación e innovación educativos. Galardonado con el Global Teacher Awards. ORCID ID: https://orcid.org/O000-0002-3177-2199. Email: angel.perez.pueyo@unileon.es

\section{Mercedes López-Aguado}

Profesora Titular de Universidad del Área de Métodos de Investigación y Diagnóstico en Educación de la Universidad de León. Ha participado en numerosos proyectos de investigación educativa. Sus líneas de investigación se han centrado en el aprendizaje tanto de la lectoescritura en educación primaria como en los procesos de aprendizaje en educación superior. En el ámbito de la educación formal, en la actualidad participa en un proyecto sobre las competencias de los docentes de los niveles educativos no universitarios. Ha dirigido varias tesis doctorales, numerosos Trabajos Fin de Grado y Finde Máster y tiene diversas publicaciones relacionadas con las temáticas anteriormente citadas. ORCID ID: https://orcid.org/0000-0002-4822-6901. Email: mmlopa@unileon.es

\section{Jesús Manso-Ayuso}

Profesor del Departamento de Pedagogía de la Universidad Autónoma de Madrid (UAM) desde 2014. Coordinador del doble grado de Maestro/a en Educación Infantil y Primaria desde 2017 y Subdirector del Programa PROMENTOR "Formación para la inclusión laboral de jóvenes con discapacidad intelectual" desde 2018. Vicedecano de Estudios de Grado de la Facultad de Formación de Profesorado y Educación de la UAM entre los años 2017 y 2020. Diplomado en Magisterio en la especialidad de Educación Especial y licenciado en Psicopedagogía. Premio Nacional a la Excelencia en el Rendimiento Académico Universitario otorgado por el Ministerio de Educación por los estudios de psicopedagogía. Máster de Mejora y Calidad de la Educación y Doctor Europeo en Educación por la UAM en 2012. ORCID ID: https://orcid.org/0000-0003-1557-3242. Email: jesus.manso@uam.es

\section{Javier Fernández-Río}

Profesor Titular de Universidad. Acreditado al cuerpo de catedráticos. Referente internacional en la implantación del aprendizaje cooperativo en Educación Física. Experto en modelos pedagógicos. Autor de diversidad de publicaciones científicas en el ámbito de la educación, la educación física y del deporte. Integrante de varios grupos de 
investigación e innovación. Investigador principal y participante de varios proyectos de investigación europeos, nacionales y autonómicos. ORCID ID: https://orcid.org/0000o002-1368-3723.Email: javier.rio@uniovi.es 